\title{
RESEARCH HIGHLIGHT The unfolding body plan of primate embryos in culture
}

\author{
Jitesh Neupane ${ }^{1,2}$, Frederick C. K. Wong ${ }^{1,2}$ and M. Azim Surani $\mathbb{D}^{1,2}$ \\ Cell Research (2020) 30:103-104; https://doi.org/10.1038/s41422-019-0269-x
}

\begin{abstract}
Improved culture of non-human primate embryos reveals the establishment of the crucial framework for subsequent development of bodily tissues and the germline in fetuses, bringing us closer to comprehending the elusive development of early human embryos.
\end{abstract}

Most of what we know about very early mammalian development after blastocyst implants in the womb comes from mouse studies. ${ }^{1}$ Little is known about development in primates, and even less so for human development, because of many challenges and constraints on studies on early human embryos. Accordingly, studies on non-human primate embryos might provide information relevant to human development. Two recent studies now report on the development of cynomolgus monkey embryos for 20 days post fertilization, and their progress through gastrulation in culture. ${ }^{2,3}$ They compare their observations on embryonic development in culture by referring to a previous study on the developing monkey embryos in vivo. ${ }^{4}$

Shortly after implantation, the inner cell mass (ICM) of humans and non-human primates develop into epithelial cells, followed by the formation of a lumen, which gives rise to the amniotic cavity (Fig. 1). The embryonic tissues themselves develop as a bilaminar disc comprising the epiblast on top of the hypoblast; the latter originates from the primitive endoderm. The epiblast and amnion epithelium encapsulate the amniotic cavity while the hypoblast endoderm and extraembryonic mesoderm encapsulate the primary yolk sac. The formation of a primitive streak along the midline of the epiblast disc marks the onset of gastrulation. Gastrulation is a pivotal event during early development when the three primary germ layers, endoderm, ectoderm, and mesoderm, appear for the first time. Primordial germ cells (PGCs), which eventually develop into sperm or eggs, also emerge around this time, either in the amnion according to some studies, epiblast or in both tissues. ${ }^{5-7}$ The anterior and posterior ends of the embryos now become distinguishable before the germ layers undergo further differentiation towards organogenesis, in which the anterior cells, e.g., develop into neuronal cells.

In the recent studies on the cynomolgus monkey embryos, Ma et al. ${ }^{2}$ and Niu et al. ${ }^{3}$ adopted in vitro culture methods previously used to culture mammalian embryos. These studies reveal aspects of non-human primate embryo development after embryo implantation, through gastrulation. These independent studies showed the development of cynomolgus monkey embryos up to 20 days post fertilization (dpf). Niu et al. used the culture system described elsewhere. ${ }^{8}$ In contrast, Ma et al. added some novel aspects to their culture conditions containing different serum concentrations, which was first optimized using mouse blastocysts. ${ }^{2}$

Broadly speaking, the development of the monkey embryos in both studies recapitulated key developmental events previously described for embryonic development in vivo., ${ }^{4,6}$ They observed the formation of the bilaminar disc structure comprising amniotic and yolk sac cavities and the establishment of an anteriorposterior axis. They also saw the emergence of the primitive streak, and the appearance of the PGC-like cells and of the structures resembling neural plate folding. ${ }^{2}$ In the future, it will be essential to conduct a rigorous, detailed analysis of development using approaches such as lineage tracing and live imaging to establish the precise origin and destiny of diverse cell types in the embryo, at a time when the embryo undergoes extensive morphogenetic changes.

For now, both studies opted to conduct single-cell transcriptome analysis in the hope to characterize the diverse cell types present in the developing embryos. Ma et al. and Niu et al. compared different stages of embryo development, which they compared with the single-cell transcriptome analysis of cynomolgus monkey embryos in vivo described previously. ${ }^{4}$ Although cells of the majority of embryonic and extraembryonic lineages found in vivo were detected in embryos in culture, some disparities in the clustering of cell types were apparently observed. The discrepancies might be due to the appearance of transient and intermediate cell lineages, but the presence of aberrant cells in embryos in culture cannot be excluded. Nonetheless, Ma et al. were able to annotate early (11-14 dpf) and late (16-17 dpf) gastrulating or amniotic cells, where the transcriptional profile of amniotic cells in embryos were not reported before. ${ }^{4}$ Niu et al., on the other hand, investigated the chromatin accessibility of the post-implantation embryo at the single-cell level, which, for example, can detect enhancer elements in different cell types during development. Overall, the cell identities from these two studies overlap with those found in vivo, suggesting that embryos in culture broadly follow events observed in vivo. Note that the culture methods used in these studies can support up to $22 \%$ of embryos to the gastrulation stage without feto-maternal interactions. Whether the efficiency of development observed reflects inherent differences in the quality of the embryos or a consequence of culture conditions is unknown. If it is the latter, further optimization of culture conditions, might result in better development at a higher efficiency.

These studies have strengthened our current understanding of the early post-implantation development in primates. They will enhance the technological and conceptual advancement for translating the knowledge to stem cell research and developmental biology. Reliable and efficient culture models could be used to investigate the morphological, molecular, and physiological properties of primate embryos, which could, in principle, be used to study early human peri- to post-implantation development in vitro. There are species-specific differences between early

\footnotetext{
${ }^{1}$ Wellcome Trust Cancer Research UK Gurdon Institute, University of Cambridge, Cambridge CB2 1QN, UK and ${ }^{2}$ Physiology, Development and Neuroscience Department, University of Cambridge, Cambridge, UK

Correspondence: M. Azim Surani (a.surani@gurdon.cam.ac.uk)
}

Published online: 8 January 2020 

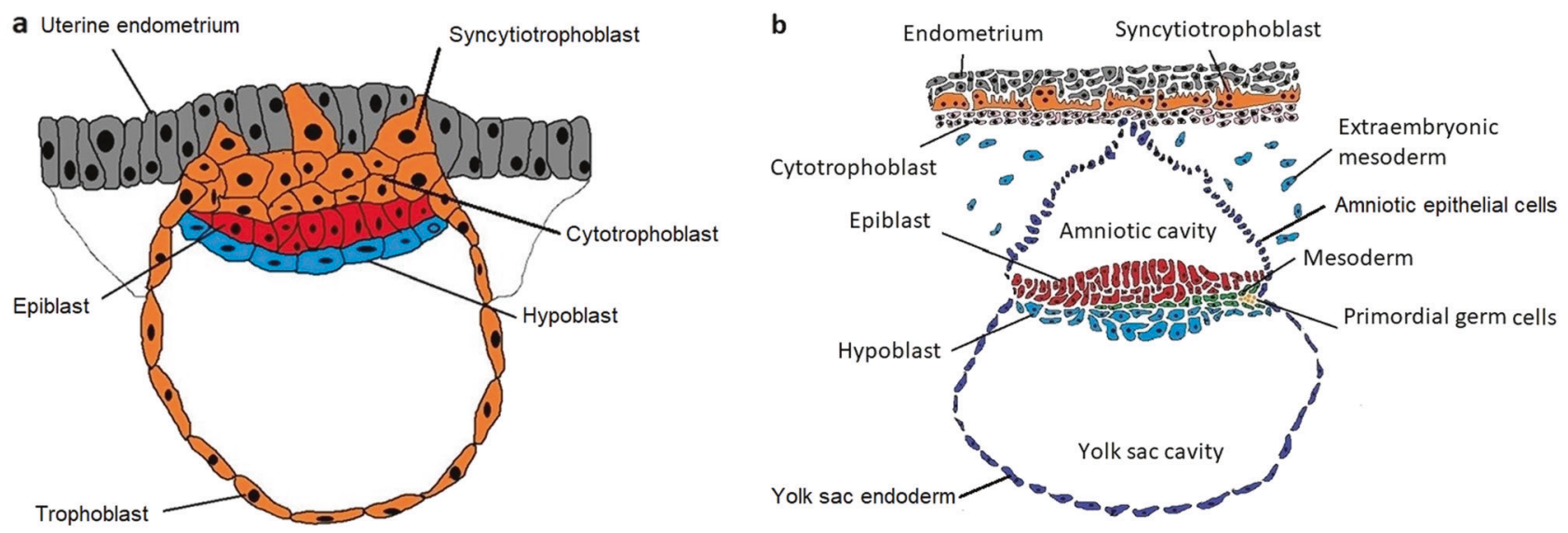

Fig. 1 Schematic illustration showing that post-implantation embryo development of primates might be relevant to humans. a Implantation begins with the attachment of blastocyst to the maternal uterine endometrium, when trophoblast cells proliferate into cytotrophoblast and syncytiotrophoblast, which eventually expand and surround the embryo. $\mathbf{b}$ The amniotic cells develop from epiblast cells and form an amniotic cavity, whereas cells from hypoblast form yolk sac endometrium, resulting in a yolk sac cavity. The embryo develops from bilaminar disc epiblast on top of hypoblast cells. Primitive streak appears at the posterior end of embryonic disc, when epiblast starts invading towards hypoblast, thus marking the initiation of gastrulation. Proliferating epiblast cells along the streak migrate to the space between epiblast and hypoblast giving rise to mesoderm cells, thereby converting bilaminar embryonic disc into a trilaminar disc. Primordial germ cells, the precursors of male and female gametes, might originate from the amnion, the epiblast or both tissues during the pregastrulation period. They migrate on the wall of the yolk sac close to the allantois, and subsequently along the hindgut before and after colonizing the gonadal ridge. (Figure adapted from Heuser et al., ${ }^{12}$ and Boroviak and Nichols ${ }^{13}$ ).

human and non-human primate development, indicating caution when considering extrapolation to human development. The differences include the timing of implantation and amniogenesis between cynomolgous monkey and humans, ${ }^{9}$ which reflects possible variations due to species evolution. ${ }^{10}$ The schedules of gastrulation and establishment of PGCs might also differ, although the regulatory network for PGC specification is apparently broadly conserved in mammals with bilaminar disc embryos. ${ }^{7,10}$ Some studies, for example, suggest that PGC-like cells in monkeys can be found in the nascent amnion during the mid-second week ( $11 \mathrm{dpf})$ of development; ${ }^{6}$ further work is needed to determine whether amnion and/or epiblast could be the site for the origin of PGCs. Note that PGC specification in the porcine bilaminar disc embryos occurs in the epiblast where the gene regulatory network is more like that in human and not mouse embryos. ${ }^{5}$ While the developmental events might be broadly similar amongst mammals, there are more significant differences in the development of the extraembryonic tissues. Combined with possible timing differences amongst mammalian species and their extraembryonic tissues, differences in the molecular mechanism of embryonic development amongst primates cannot be excluded.

The recent studies on gastrulating non-human primate embryos in culture represent a significant advance; much will, however, depend on whether the culture models can be used for more in-depth mechanistic studies. While extrapolation from these studies to early human development is possible, crucial species differences amongst primates cannot be entirely excluded. With legal restrictions on similar studies on human embryos, alternative approaches such as establishing in vitro models for early human development with embryonic stem cells, and generating embryoid-like structures is an option. ${ }^{11}$ Increasing improvements and sophistication of the in vitro models might for now address some key questions but cannot substitute entirely for direct studies on very early human development. Such studies, if possible, are of potential value for advances in regenerative medicine and treatment of human diseases.

\section{REFERENCES}

1. Rossant, J. \& Tam, P. P. L. Science 360, 1075 (2018).

2. Ma, H. et al. Science 366, eaax7890 (2019).

3. Niu, Y. et al. Science 366, eaaw5754 (2019).

4. Nakamura, T. et al. Nature 537, 57-62 (2016).

5. Kobayashi, T. et al. Nature 546, 416-420 (2017).

6. Sasaki, K. et al. Dev. Cell 39, 169-185 (2016).

7. Irie, N. et al. Cell 160, 253-268 (2015).

8. Deglincerti, A. et al. Nature 533, 251-254 (2016).

9. Luckett, P. Am. J. Anat. 144, 149-167 (1975).

10. Sybirna, A., Wong, F. C. K. \& Surani, M. A. Curr. Top. Dev. Biol. 135, 35-89 (2019).

11. Zheng, Y. et al. Nature 573, 421-425 (2019).

12. Heuser, C. H. \& Streeter, G. L. Carnegie Inst. Wash. Publ. 525, 15-55 (1941).

13. Boroviak, T. \& Nichols, J. Development 144, 175-186 (2017). 